\title{
ФАЗОЧАСТОТНОЕ ОБНАРУЖЕНИЕ СЕЙСМИЧЕСКИХ СИГНАЛОВ
}

\author{
Кочегуров Александр Иванович 1 , \\ kaicc@tpu.ru
}

\author{
Виктор Герингер, \\ geringer@dhbw-ravensburg.de \\ 1 Национальный исследовательский Томский политехнический университет, \\ Россия, 634050, г. Томск, пр. Ленина, 30. \\ 2 Баден-Вюртембергский объединенный государственный университет, \\ Германия, DHBW-Равенсбург, Marienplatz, 2.
}

\begin{abstract}
Актуальность. В настоящее время при поиске нефтяных и газовых месторождений большое внимание уделяется более активному вовлечению в разведочный процесс слабо исследованных территорий, а также доисследованию природных резервуаров в районах промышленного освоения месторождений. Для этих иелей широко применяются методы сейсморазведки, в результате чего формируются огромные массивы данных, подлежащие последующей обработке и интерпретации. Одной из задач, решаемой на этапе обработки, является надежное обнаружение сейсмических сигналов, особенно важное значение эта задача имеет при прослеживании волн, так как от достоверности обнаружения зависит качество построения отражающих границ. Поэтому построение эффрективных методов и алгоритмов обнаружения сигналов на основе анализа ФЧХ, обладающих более высокой степенью устойчивости к помехам, является весьма актуальной задачей.

Цель: на основе оптимальной и субоптимальной обработки ФЧХ сейсмических волн, получаемых при поиске нефртяных и газовых месторождений, построить методы обнаружения сигналов и исследовать их эфрфективность на моделях сейсмоимпульсов путем вычисления вероятностных характеристик ошибок обнаружения.

Методы и средства исследования: методы статистического анализа и синтеза алгоритмов, теория случайных процессов, дискретное преобразование Фурье, методы обработки и интерпретации сейсмических данных, математическое моделирование и вычислительный эксперимент.

Результаты. На основе метода максимального правдоподобия построена оптимальная процедура обнаружения сейсмических сигналов по их фразочастотным характеристикам. Получены аналитические выражения для вероятностей ошибок обнаружения. Показано, что оптимальное фразочастотное обнаружение обеспечивает результаты, близкие к результатам абсолютно оптимального метода обнаружения сигналов. Для практической реализации разработанного метода предложена субоптимальная обработка ФЧХ (равновесная обработка), не требующая инфрормации о распределении энергии сигнала в частотной области. Проведены исследования помехоустойчивости метода обнаружения с равновесной обработкой, и получены оценки потерь в зависимости от числа использованных частотных компонент в спектре сигнала. Показано место применения разработанных методов обнаружения при решении задач прослеживания сейсмических волн.
\end{abstract}

\section{Ключевые слова:}

Сейсмические волны, оптимальное фазочастотное обнаружение, отношение правдоподобия, равновесная обработка, прослеживание сейсмических волн

\section{Введение}

Обнаружение отраженных сейсмических волн является одной из важнейших задач, решаемых при обработке и интерпретации данных, получаемых при поиске нефтяных и газовых месторождений $[1,2]$. Ocобое место эта задача занимает при построении алгоритмов прослеживании сейсмических волн, так как процесс прослеживания волн в обязательном порядке включает обнаружение фиксированных волн и определение их временного положения [1].

В современных комплексах обработки сейсмической информации при прослеживании волн применяются алгоритмы, разработанные по схеме прослеживания энергетических аномалий сейсмических сигналов [3, 4], поэтому и обнаружение волн, как правило, осуществляется на основе сравнения с порогом суммарной мощности сигнала, выделяемого с помощью амплитудных критериев. При этом наиболее часто применяется корреляционный приемник или согласованная фильтрация $[1,2]$. В случае исследования тонкослоистых сред для выделения сиг- налов в зонах повышенной интерференции в настоящее время широко применяются методы деконволюции [5-8], в которых также в основном используются энергетические параметры сигнала, при этом фазовые спектры не вычисляются, их просто принимают в виде минимально фазовых или нуль-фазовых функций [9-13]. В то же время в сложных сейсмогеологических условиях, например, при обнаружении сигналов, когда отношение сигнала к шуму мало, или наблюдаются зоны интерференции волн, фазочастотные характеристики (ФЧХ) являются более устойчивыми по сравнению с энергетическими параметрами сигналов. Кроме того, в сложный закон изменения ФЧХ заложена информация, на основе которой удается выделять слабые сигналы и производить оценку их временного положения [14]. Также фазовые спектры дают возможность оценить поглощение геологических сред, характер напластования, акустические свойства и толщину слоев, что может служить дополнительными признаками при обнаружении сигналов. 
К сожалению, до настоящего времени, использование ФЧХ отраженных волн для обнаружения сигналов не нашло широкого применения на практике, что связано прежде всего с особенностями и неоднозначностью вычисления ФЧХ сейсмических волн, а также недостаточной изученностью их взаимосвязи с конфигурацией границ и параметрами геологического разреза. В то же время существуют примеры весьма успешного и эффективного использования ФЧХ отраженных волн для определения временного положения сигналов при решении задач прослеживания в тонкослоистых средах $[14,15]$, что позволяет говорить о целесообразности применения ФЧХ для обнаружения фиксированных волн.

Таким образом, методы обнаружения, основанные на оптимальной обработке фазовых спектров сейсмических волн, могут оказаться весьма эффективным инструментом для уменьшения ошибок обнаружения при выделении слабых сигналов. Поэтому разработка, исследование и применение методов фазочастотного обнаружения сейсмических волн, в том числе в алгоритмах прослеживания, имеет важное значение и является весьма актуальной задачей.

\section{Постановка задачи}

Предположим, что наблюдается участок сейсмической трассы $x(t)$, который может содержать аддитивную смесь сигнала $s(t)$ и помехи $\xi(t)$ или только помеху $\xi(t)$. Примем: $s(t)=S_{d}(T)$, где $S_{d}(T)-$ множество сигналов ограниченной длительности, основная энергия которых сосредоточена в полосе от 10 до 100 Гц. Однако, несмотря на относительно узкую полосу частот, данные сигналы относятся к классу широкополосных сигналов. $\xi(t)$ - стационарная нормально распределенная помеха с корреляционной функцией $\sigma_{\xi}^{2} R_{\xi}(\tau)$.

Задача состоит в том, чтобы построить процедуру обнаружения сигналов, когда оптимальной обработке подвергается только ФЧХ анализируемого участка сейсмотрассы.

\section{Оптимальное фазочастотное обнаружение сейсмических сигналов}

Пусть функции $\xi(t), s(t)$ и $x(t)=s(t)+\xi(t)$ на интервале времени Т могут быть представлены в частотной области $\Omega$ своими Фурье-образами. Тогда для построения оптимального фазового критерия обнаружения сигнала используем метод максимального правдоподобия на основе анализа ФЧХ. Для реализации данного метода сформируем отношение правдоподобия в виде [16]:

$$
L(\Delta \varphi)=\frac{W_{m}\left(\Delta \varphi\left(\omega_{1}\right), \Delta \varphi\left(\omega_{2}\right), \ldots, \Delta \varphi\left(\omega_{m}\right) \mid H_{1}\right)}{W_{m}\left(\varphi_{\xi}\left(\omega_{1}\right), \varphi_{\xi}\left(\omega_{2}\right), \ldots, \varphi_{\xi}\left(\omega_{m}\right) \mid H_{0}\right)},
$$

где $\quad W_{m}\left(\Delta \varphi\left(\omega_{1}\right), \Delta \varphi\left(\omega_{2}\right), \ldots \Delta \varphi\left(\omega_{m}\right) \mid H_{1}\right) \quad$ - $m$-мерная плотность вероятности отклонения ФЧХ смеси от ФЧХ сигнала в предположении, что верна гипотеза $H_{0}$ (сигнал есть); $W_{m}\left(\varphi_{\xi}\left(\omega_{1}\right), \Delta \varphi_{\xi}\left(\omega_{2}\right), \ldots \Delta \varphi_{\xi}\left(\omega_{m}\right) \mid H_{0}\right)-$ $m$-мерная плотность вероятности ФЧХ помехи в предположении, что верна гипотеза $H_{0}$ (сигнала нет);

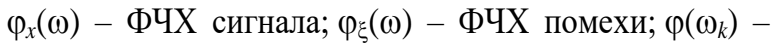
ФЧХ смеси сигнала с помехой; $\Delta \varphi(\omega)=\varphi_{x}(\omega)-\varphi_{s}(\omega)-$ отклонение ФЧХ смеси от ФЧХ сигнала; $m=\frac{\Omega}{\Delta \omega}-$ число спектральных компонент; $\Delta \omega$ - шаг дискретизации по частоте.

Теперь, если принять, что $\mid \varphi_{x}\left(\omega \mid \leq \pi\right.$ и $\mid \varphi_{s}(\omega \mid \leq \pi$, одномерная плотность вероятности значений ФЧХ помехи равна $W\left(\varphi_{\xi}\left(\omega_{k}\right)\right)=\frac{1}{2 \pi}$, а $\varphi_{x}\left(\omega_{k}\right)$ совпадает с угловой координатой вектора $\left(\rho \cos \varphi_{x}\left(\omega_{k}\right), \rho \sin \varphi_{x}\left(\omega_{k}\right)\right)$. В этом случае отношение правдоподобия (1) для независимых значений $\Delta \varphi\left(\omega_{k}\right)$ имеет вид [2]:

$$
\begin{aligned}
& L(\Delta \varphi)= \\
& =\prod_{k=1}^{m}\left\{\begin{array}{l}
\exp \left(-\frac{\delta^{2}\left(\omega_{k}\right)}{2}\right)+\sqrt{2 \pi} \delta\left(\omega_{k}\right) \times \\
\cos \left(\Delta \varphi\left(\omega_{k}\right)\right) \Phi\left[\delta\left(\omega_{k}\right) \cos \left(\Delta \varphi\left(\omega_{k}\right)\right)\right] \times \\
\times \exp \left(-\frac{\delta^{2}\left(\omega_{k}\right)}{2} \sin \left(\Delta \varphi\left(\omega_{k}\right)\right)\right.
\end{array}\right\},
\end{aligned}
$$

где $\Phi$ - преобразование Лапласа; $k$ - номер частотной компоненты, $k=\overline{1, m ;} \delta^{2}\left(\omega_{k}\right)$ - отношение энергии сигнала к дисперсии помехи на частоте $\omega_{k}$, которое определяется как:

$$
\delta^{2}\left(\omega_{k}\right)=\frac{A_{s}^{2}\left(\omega_{k}\right)}{\sigma^{2}\left(\omega_{k}\right)},
$$

где $A_{s}\left(\omega_{k}\right)$ - величина АЧХ сигнала на частоте $\omega_{k}$; $\sigma^{2}\left(\omega_{k}\right)$ - дисперсия, пропорциональная спектральной плотности помехи на частоте $\omega_{k}$.

Тогда по критерию максимального правдоподобия принимается решение о том, что «сигнал есть», если

$$
L(\Delta \varphi)>C,
$$

и принимается решение о том, что «сигнала нет», если

$$
L(\Delta \varphi)<C .
$$

Прологарифмируем выражение (2) и получим:

$$
\ln L(\Delta \varphi)=
$$

$$
=\sum_{k=1}^{m} \ln \left\{\begin{array}{l}
\exp \left(-\frac{\delta^{2}\left(\omega_{k}\right)}{2}\right)+\sqrt{2 \pi} \delta\left(\omega_{k}\right) \times \\
\cos \left(\Delta \varphi\left(\omega_{k}\right)\right) \Phi\left[\delta\left(\omega_{k}\right) \cos \left(\Delta \varphi\left(\omega_{k}\right)\right)\right] \times \\
\times \exp \left(-\frac{\delta^{2}\left(\omega_{k}\right)}{2} \sin ^{2}\left(\Delta \varphi\left(\omega_{k}\right)\right)\right.
\end{array}\right\} .
$$

Зная монотонный характер логарифмической функции, можно утверждать: принимается решение «сигнал есть», если $\ln L(\Delta \varphi)>\ln C$, и принимается решение о том, что «сигнала нет», если $\ln L(\Delta \varphi)<\ln C$.

Далее последовательно рассмотрим два частных случая: сигнал сильный и сигнал слабый. При сильном сигнале $\left(\delta\left(\omega_{k}\right)>1\right)$, величины $\Delta \varphi\left(\omega_{k}\right)$ подчиняются нормальному закону распределения: 


$$
W\left(\Delta \varphi\left(\omega_{k}\right)\right)=\frac{1}{2 \pi \sigma_{\varphi_{x}}\left(\omega_{k}\right)} \exp \left(-\frac{\Delta \varphi^{2}\left(\omega_{k}\right)}{2 \sigma_{\varphi_{x}}^{2}\left(\omega_{k}\right)}\right) .
$$

В этом случае, если принять величину порога $C=1$ (критерий идеального наблюдателя) [16, 17], то, переходя к выборке с объемом $m=\Omega / \Delta \omega$, нетрудно показать, что решающее правило (3) имеет вид

$$
\sum_{k=1}^{m} \frac{\Delta \varphi^{2}\left(\omega_{k}\right)}{2 \sigma_{\varphi_{x}}^{2}\left(\omega_{k}\right)} \leq \sum_{k=1}^{m} \ln \frac{\sqrt{2 \pi}}{\sigma_{\varphi_{x}}\left(\omega_{k}\right)} .
$$

В случае слабого сигнала $\left(\delta\left(\omega_{k}\right) \leq 1\right) m$-мерная плотность вероятности отклонения ФЧХ смеси от ФЧХ сигнала без учета членов с более высокими степенями, чем $\delta^{2}\left(\omega_{k}\right)$, имеет вид:

$$
=\frac{1}{2 \pi}\left(\begin{array}{c}
W_{m}\left(\Delta \varphi\left(\omega_{k}\right)\right)= \\
1+\delta\left(\omega_{k}\right) \sqrt{\frac{\pi}{2}} \cos \left(\Delta \varphi\left(\omega_{k}\right)\right)+ \\
+\frac{\delta^{2}\left(\omega_{k}\right)}{2} \cos \left(2 \Delta \varphi\left(\omega_{k}\right)\right)
\end{array}\right) .
$$

При этом дисперсия флуктуаций значений ФЧХ составляет:

$$
\delta^{2}\left(\omega_{k}\right)=\frac{\pi^{2}}{3}-\sqrt{2 \pi} \delta\left(\omega_{k}\right) .
$$

Тогда логарифм отношения правдоподобия (3) для слабого сигнала запишется:

$$
\begin{gathered}
\ln L(\Delta \varphi)= \\
=-\sum_{k=1}^{m} \frac{\pi}{8} \delta^{2}\left(\omega_{k}\right)+\sum_{k=1}^{m} \sqrt{\frac{\pi}{2}} \delta\left(\omega_{k}\right) \cos \left(\Delta \varphi\left(\omega_{k}\right)\right) .
\end{gathered}
$$

Принимается решение «сигнал есть», если

$$
-\sum_{k=1}^{m} \frac{\pi}{8} \delta^{2}\left(\omega_{k}\right)+\sum_{k=1}^{m} \sqrt{\frac{\pi}{2}} \delta\left(\omega_{k}\right) \cos \left(\Delta \varphi\left(\omega_{k}\right)\right) \geq C .
$$

При $C=1$ данное выражение перепишется в виде

$$
\sum_{k=1}^{m} \sqrt{\frac{\pi}{2}} \delta\left(\omega_{k}\right) \cos \left(\Delta \varphi\left(\omega_{k}\right)\right) \geq \sum_{k=1}^{m} \frac{\pi}{8} \delta^{2}\left(\omega_{k}\right) .
$$

На первый взгляд отличия между выражениями (4) и (5), определяющими фазочастотное обнаружение сильного и слабого сигнала, довольно существенны, однако если учесть, что при сильном сигнале флюктуации ФЧХ незначительны

$$
\cos \left(\Delta \varphi\left(\omega_{k}\right)\right) \approx 1-\frac{1}{2} \Delta \varphi^{2}\left(\omega_{k}\right) .
$$

В то же время $\delta^{2}\left(\omega_{k}\right)=\frac{1}{\sigma_{\varphi_{x}}^{2}\left(\omega_{k}\right)}$. Тогда нетрудно увидеть, что алгоритм фазочастотного обнаружения слабых сигналов будет оптимальным и для сильных сигналов, но не наоборот.

Таким образом, выражение (5) в принятом смысле определяет структуру алгоритма оптимального фазочастотного обнаружения сейсмических сигналов с известными параметрами. Для его реализации необходимо:

1) вычислить косинусы разности между значениями ФЧХ смеси и ФЧХ сигнала для всех $\omega \in \Omega$;

2) просуммировать все полученные величины косинусов, причем каждую с весом, пропорциональным отношению сигнал/шум на данной частоте;

3) сравнить полученное значение $L(\Delta \varphi)$ с порогом, в общем случае зависящим от отношения энергии сигнала к спектральной плотности шума, формы АЧХ сигнала и спектральной плотности шума.

Рассмотрим теперь ошибки, свойственные оптимальному фазочастотному обнаружению слабого сигнала. Для этого найдем вероятности ложного обнаружения $P\left(H_{1} \mid 0\right)$ и вероятности пропуска сигнала $P\left(H_{0} \mid C\right)$. Принимая, что размер выборки достаточно большой $(m>>1)$, воспользуемся асимптотической нормальностью левой части выражения (5) и получим:

$$
\begin{aligned}
& P\left(H_{1} \mid 0\right)=1-\Phi\left(\frac{\sqrt{\pi}}{4} \sum_{k=1}^{m} \delta^{2}\left(\omega_{k}\right)\right) ; \\
& P\left(H_{0} \mid C\right)=1-\Phi\left(\frac{\sqrt{\pi}}{4} \sum_{k=1}^{m} \delta^{2}\left(\omega_{k}\right)\right) .
\end{aligned}
$$

Тогда величина полной вероятности ошибки составит:

$$
\begin{aligned}
P_{\text {ош }} & =0,5\left[P\left(H_{1} \mid 0\right)+P\left(H_{0} \mid C\right)\right]= \\
& =1-\Phi\left(\frac{\sqrt{\pi}}{4} \sum_{k=1}^{m} \delta^{2}\left(\omega_{k}\right)\right) .
\end{aligned}
$$

Для сравнения с абсолютно-оптимальным методом обнаружения (т. е. когда оптимально обрабатывается и АЧХ и ФЧХ смеси) приведем полную вероятность ошибки корреляционного приемника [18]:

$$
P_{\text {ош }}=1-\Phi\left(\frac{\sqrt{\sum_{k=1}^{m} \delta^{2}\left(\omega_{k}\right)}}{2}\right) .
$$

Анализ выражений (6) и (7) показывает, что оптимальное фазочастотное обнаружение дает несколько худшие результаты, чем абсолютно-оптимальное, однако полученная разница невелика. Таким образом, основная информация о слабом сигнале заложена в его ФЧХ.

\section{Фазочастотное обнаружение}

с равновесной обработкой

Реальные условия возбуждения, распространения и регистрации сейсмических сигналов приводят, как правило, к необходимости решения задачи обнаружения в условиях априорной неопределенности. Эта неопределенность обычно выражается в незнании формы сигнала, а следовательно и распределение энергии сигнала по частоте становится неизвестным. Поэтому примем, что все значения $Ф Ч Х$ в выражении (5) суммируются с одинаковыми весами. При равновесной обработке с порогом сравнивается величина 


$$
\ln L_{1}(\Delta \varphi)=\sqrt{\frac{\pi}{2}} \sum_{k=1}^{m_{0}} \cos \left(\Delta \varphi\left(\omega_{k}\right)\right)
$$

где $m_{0}<m$.

Для оценки качества обнаружения в соответствии с (8) сравним величины суммарного отношения сигнал/помеха $q_{\Sigma}^{\prime}$ и $q_{\Sigma}^{\prime \prime}$, рассчитанные соответственно для (5) и (8).

Из (5) следует, что при оптимальном фазочастотном обнаружении

$$
q_{\Sigma}^{\prime}=\frac{\sqrt{\pi}}{4} \sqrt{\sum_{k=1}^{m} \delta^{2}\left(\omega_{k}\right)} .
$$

Для оценки $q_{\Sigma} "$ проведем анализ выражения (8) и получим:

$$
q_{\Sigma}^{\prime \prime}=\frac{\sqrt{\pi}}{4 \sqrt{m_{0}}} \sum_{k=1}^{m_{0}} \delta\left(\omega_{k}\right) .
$$

Тогда потери за счет равновесности обработки могут быть охарактеризованы следующим параметром [19]:

$$
\eta=\left(\frac{q_{\Sigma}^{\prime}}{q_{\Sigma}^{\prime \prime}}\right)^{2}-1 .
$$

В [19] предложена методика оценки потерь $\eta$ равновесной обработки многочастотного сигнала при абсолютно-оптимальном (корреляционном) алгоритме обнаружения. Используем эту методику для оценки потерь при фазочастотном обнаружении. Будем считать, что частотный диапазон анализа $\Omega$ задан так, что $\delta\left(\omega_{k+1}\right) \leq \delta\left(\omega_{k}\right), k=1,2, \ldots, m-1$.

Тогда на основании принятой методики нетрудно показать, что максимальные потери в соответствии с выражением (9) составляют:

$$
\eta_{\max }=\sum_{k=1}^{m}(\sqrt{k}-\sqrt{k-1})^{2}-1
$$

Тогда максимальная величина полной вероятности ошибки при равновесной обработке с учетом (10) определяется выражением:

$$
P_{\text {ош }}=1-\Phi\left(\frac{\sqrt{\pi}}{4} \sqrt{\frac{\sum_{k=1}^{m} \delta^{2}\left(\omega_{k}\right)}{\sum_{k=1}^{m}(\sqrt{k}-\sqrt{k-1})^{2}}}\right) .
$$

Из сравнения (7) и (11) следует, что с ростом $m$ при равновесной обработке ошибки обнаружения увеличиваются. Так, в предположении, что $q_{\Sigma}(m)=$ const при $m=2, \eta_{\max }=0,17, m=3, \eta_{\max }=0,27$, $m=3, \eta_{\max }=0,58, m=20, \eta_{\max }=0,754$. Однако, несмотря на это, даже при $m=20$ надежность обнаружения достаточно высока.

Таким образом, применение равновесного суммирования при реализации алгоритма фазочастотного обнаружения сейсмических сигналов не приводит к существенным потерям. При этом не нужно знать распределение отношения сигнал/помеха по частотам, а достаточно оценить только суммарное отношение сигнал/помеха на сейсмической трассе. Для практического использования предлагаемого метода можно выбрать одну из методик расчета суммарного отношения сигнал/помеха, применяемых в ряде известных процедур обработки сейсмических данных [20] или, например, методику расчета, предложенную при детектировании изображений в [21].

\section{Выбор полосы частот при фазочастотном обнаружении сейсмических сигналов}

Принципиальное отличие алгоритма фазочастотного обнаружения широкополосных сигналов от алгоритма фазового обнаружения узкополосных сигналов заключается в том, что здесь результаты обнаружения существенно зависят от ширины спектра $\Omega$ (числа гармоник $m$ ), формы АЧХ сигнала и формы спектральной плотности $N(\omega)$, а следовательно, появляется дополнительная возможность повышения надежности обнаружения путем рационального выбора полосы частот $\Omega$.

Будем считать, что помеха имеет равномерную спектральную плотность в полосе частот $\Omega$ (белый шум), т. е. $\sigma^{2}(\omega)=\sigma^{2}=\frac{N_{0}}{T}$. Тогда, подставляя $\sigma^{2}$ в (6), получим известное выражение полной вероятности ошибки при фазовом обнаружении слабого узкополосного сигнала [16]:

$$
P_{\text {ош }}=1-\Phi\left(\sqrt{\frac{\pi}{8} \cdot \frac{\varepsilon_{\mathrm{c}}}{N_{0}}}\right),
$$

где $\varepsilon_{\mathrm{c}}-$ энергия сигнала; $N_{0}$ - спектральная плотность белого шума.

Рассмотрим, как изменяется величина полной вероятности ошибки в зависимости от числа учитываемых гармоник $m$ в спектре сигнала, формы АЧХ сигнала и вида $N(\omega)$. Сначала примем, что сигнал имеет равномерную АЧХ в полосе $\Omega\left(A_{s}(\omega)=A\right.$ для $\left.\omega \in \Omega\right)$, помеха - белый шум. Тогда (12) запишется

$$
P_{\text {ош }}=1-\Phi\left(\sqrt{\frac{\pi}{8} \cdot \frac{A_{s}^{2}}{N_{0} / m}}\right) .
$$

Считая, что энергия сигнала не меняется, будем увеличивать $m$ (или, что адекватно, расширять $\Omega$ ). В этом случае ширина спектра помехи тоже растет и, следовательно, спектральная плотность $N_{0} / m$ в выражении (13) уменьшается.

На рис. 1 показаны кривые полной вероятности ошибки в зависимости от отношения сигнал/помеха при различных $m$. Из рисунка видно, что расширение полосы частот позволяет существенно понизить ошибки обнаружения, а следовательно, дает возможность выделить слабые сигналы с высокой достоверностью. При этом энергия сигнала не мала, она просто распределена по частотной области так, что в каждой точке этой области мала её мощность. 


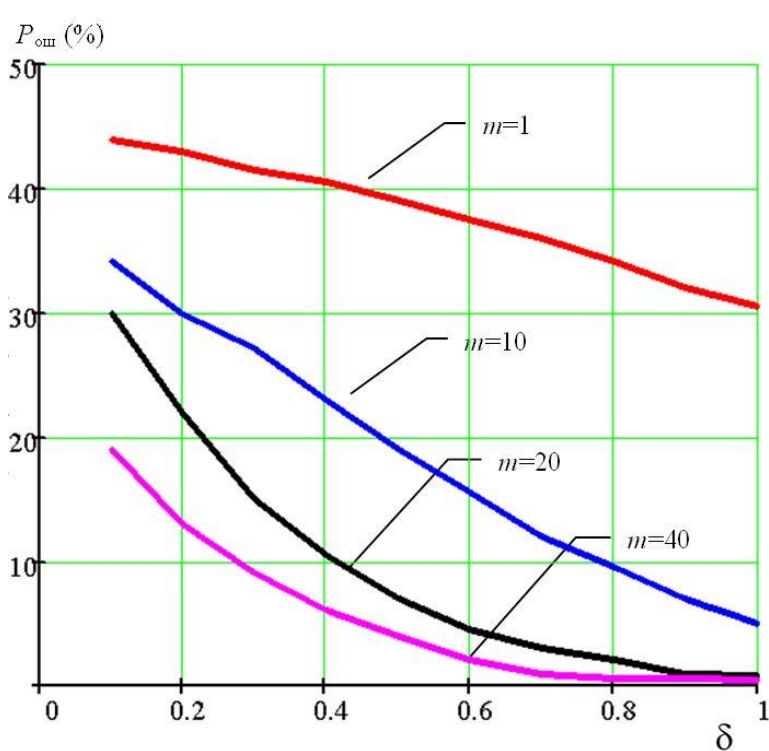

Рис. 1. Вероятность ошибок при обнаружении сигналов с равномерной АЧХ по оценке их ФЧХ для различных $m$

Fig. 1. Probability of errors when detecting signals with uniform frequency response according to their phase response for various $m$

Рассмотрим влияние формы АЧХ сигнала на величину $P_{\text {ош }}$ на примере импульса с колокольной огиба-

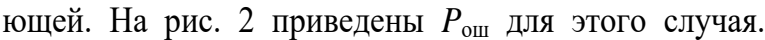
Здесь так же, как и при равномерной АЧХ, расширение $\Omega$ приводит к уменьшению ошибок обнаружения, однако разница, например, между $m=40$ и $m=20 \mathrm{не}$ такая существенная, как на рис. 1. Это связано с тем, что большая часть энергии сигнала сосредоточена в более узкой области спектра. В то же время использование одной $(m=1)$, даже самой информативной, гармоники не обеспечивает высокой надежности обнаружения.

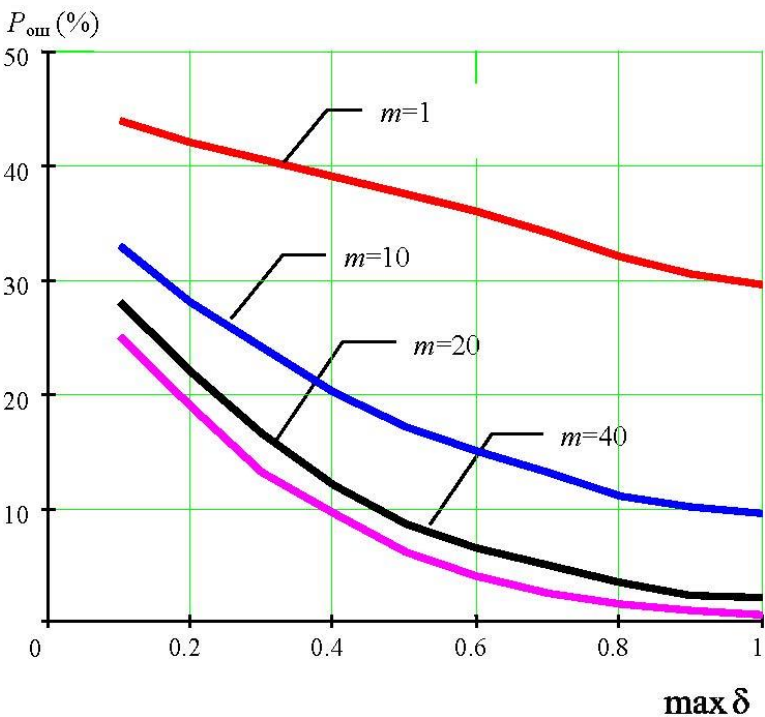

Pис. 2. Вероятность ошибок при обнаружении импульса с колокольной огибающей для различных $m$

Fig. 2. Probability of errors in detecting pulse with a bell envelope for various $m$
На рис. 3 показаны кривые $P_{\text {ош }}$ при обнаружении сигнала с равномерной АЧХ на фоне помехи с корреляционной функцией: $R_{\xi}(\tau)=\sigma_{\xi}^{2} e^{-\omega_{x} \tau} \cdot \cos \omega_{0} \tau$. Из рис. 3 следует, что при $\max \delta<0,3$ расширение $\Omega$ менее эффективно, чем при белом шуме, т. к. с увеличением $m$ увеличивается и спектральная плотность помехи.

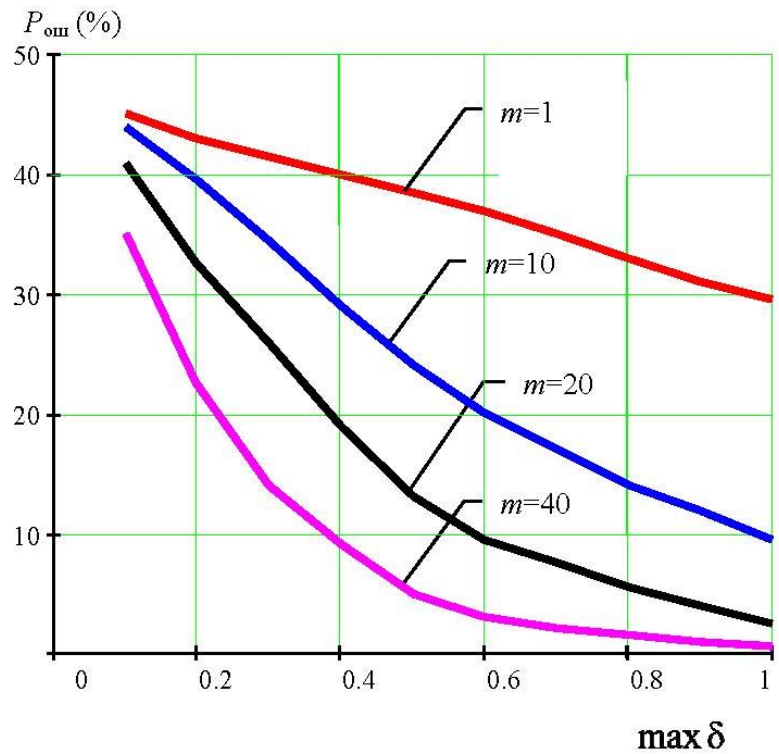

Рис. 3. Вероятность ошибок при обнаружении сигналов

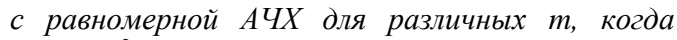
$R_{\xi}(\tau)=\sigma_{\xi}^{2} e^{-\omega_{x} \tau} \cdot \cos \left(\omega_{0} \tau\right)$

Fig. 3. Probability of errors when detecting signals with uniform frequency response for various $m$, when $R_{\xi}(\tau)=\sigma_{\xi}^{2} e^{-\omega_{x} \tau} \cdot \cos \left(\omega_{0} \tau\right)$

Таким образом, проведенный анализ показал, что выбор частотной полосы анализа оказывает существенное влияние на результаты фазочастотного обнаружения.

\section{Заключение}

На основании вышеизложенного можно говорить о том, что обнаружение сейсмических сигналов на основе оптимальной обработки их ФЧХ позволяет получать результаты, близкие к результатам абсолютно-оптимального метода обнаружения (корреляционный приемник), а в случае слабого сигнала ничем не уступающие корреляционному приему. Однако для реализации оптимального фазочастотного метода обнаружения сейсмических сигналов, как уже отмечалось, необходимо знать распределение энергии сигнала в анализируемом диапазоне частот, что при решении практических задач весьма затруднительно. Поэтому в статье предлагается и исследуется метод фазочастотного обнаружения с равновесной обработкой, который обеспечивает надежное обнаружение слабых сигналов и менее критичен к наличию априорной информации.

В то же время при фазочастотном обнаружении с равновесной обработкой особо важное значение имеет выбор частотной полосы анализа. Так, если при оптимальном фазочастотном обнаружении учет гармоник с довольно низким отношением сигнал/помеха 
не влияет в целом на результаты обнаружения, то при равновесной обработке использование этих гармоник в соответствии с (11) снижает качество обнаружения и тем сильнее, чем больше таких гармоник в спектре обнаруживаемого сигнала. Следовательно, равновесная обработка, требующая меньше априорной ин-

\section{СПИСОК ЛИТЕРАТУРЫ}

1. Боганик Г.Н., Гурвич И.И. Сейсморазведка. - Тверь: АИС, 2006. $-744 \mathrm{c}$.

2. Левянт В.Б., Квасов И.Е., Петров И.Б. Обоснование прямого обнаружения и картирования трещиноватых пластов на основе использования продольных рассеянных волн // Технологии сейсморазведки. - 2017. - № 1. - С. 72-89.

3. Денисов М.С. Алгоритм устойчивой адаптивной сигнатурной деконволюции // Технологии сейсморазведки. - 2017. - № 1. C. $48-55$.

4. Sparse blind deconvolution of seismic data via spectral projectedgradient / Naveed Iqbal, Entao Liu, J.H. McClellan, Abdullatif A. Al-Shuhail // IEEE Access. - 2019. - P. 1-12. URL: https://arxiv.org/abs/1611.03754 (дата обращения 05.03.2020).

5. Сильвиа М.Т., Робинсон Э.А. Обратная фильтрация геофизических временных рядов при разведке на нефть и газ. - М.: Недра, 1983. - 382 c.

6. Mohamed Mhmod, Feng Xuan, Xu Cong. Parameters effects on spiking deconvolution of land seismic data // Global Geology. 2015. - V. 18 (4). - P. 226-231.

7. Романов В.В. Возможности повышения разрешенности сейсмограмм метода преломленных волн (МПВ) // Технологии сейсморазведки. - 2013. - № 4. - С. 67-73.

8. Станкевич Л.С. Обработка сейсмических разрезов методом динамического сжатия // Геофизика, геофизическое приборостроение. - 2015. - № 1 (21). - С. 78-85.

9. Митрофанов Г.М. Гомоморфная фильтрация и слепая деконволюция // Технологии сейсморазведки. - 2015. - № 1. C. $46-56$

10. Egbai J.C., Atakpo E., Aigbogun C.O. Predictive deconvolution in seismic data processing in Atala prospect of rivers State, Nigeria // Advances in Applied Science Research. - 2012. - V. 3 (1). P. 520-529.

11. Lindberg D.V., More H. Blind categorical deconvolution in twolevel hidden Markov models // IEEE Transactions on Geoscience and Remote Sensing. - 2014. - V. 52. - P. 7435-7447. формации о свойствах сигналов и помех, накладывает более жесткие ограничения на выбор параметров алгоритма. Чтобы обойти эти трудности, необходимо более детально проводить качественный анализ природы сейсмических волн, регистрируемых на данной площади.

12. Idan Ram, Israel Cohen. Multichannel deconvolution of seismic signals using statistical MCMC methods // IEEE Transactions on signal processing. - 2010. - V. 58. - № 5. - P. 2757-2770.

13. Kazemi N., Bongajum E., Sacchi M. Surface-consistent sparse multichannel blind deconvolution of seismic signals // IEEE Transactions on geoscience and remote sensing. - 2016. - V. 54. P. $3200-3207$.

14. Кочегуров А.И., Иванченков В.П., Орлов О.В. Прогноз геологического разреза на основе детальной обработки сейсмических материалов в программно-алгоритмическом комплексе «Геосейф» // Известия Томского политехнического университета. Инжиниринг георесурсов. - 2019. - Т. 330. - № 6. C. $134-144$.

15. Phase/frequency deconvolution of seismic signals / A.I. Kochegurov, E.A. Kochegurova, A.E. Kozlov, A.S. Mongolin // ISSCS 2019 International Symposium on Signals, Circuits and Systems, Proceedings. - Iasi, Romania, 2019. - New York: IEEE, 2019. P. $1-4$.

16. Пестряков В.Б. Фазовые радиотехнические системы. - М.: Сов. Радио, 1968. -468 c.

17. Левин Б.Р. Теоретические основы статистической радиотехники. - М.: Сов. Радио, 1974. - Кн. 1. -552 с.

18. Левин Б.Р. Теоретические основы статистической радиотехники. - М.: Сов. Радио, 1975. - Кн. 2. -392 с.

19. Поиск, обнаружение и измерение параметров сигналов в радионавигационных системах / под ред. Ю.М. Казаринова. - М. Сов. Радио, 1975. - 296 с

20. Кац С.А., Ершова Т.Н., Шубик Б.М. Методика и результаты определения отношения сигнал/шум при регистрации сейсмического поля // Прикладная геофизика. - 1973. - Вып. 72. C. $59-70$.

21. Ein stochastischer Algorithmus zur Bildgenerierung durch einen zweidimensionalen Markoff-Erneuerungsprozess / D. Dubinin, V. Geringer, A. Kochegurov, K. Reif // at- Automatisierungstechnik. 2014. - V. 62. - № 1. - P. 57-64.

Поступила: 09.04.2020 г.

\section{Информация об авторах}

Кочегуров А.И., кандидат технических наук, доцент отделения информационных технологий Инженерной школы информационных технологий и робототехники Национального исследовательского Томского политехнического университета.

Герингер В., кандидат технических наук, заведующий лабораторией автомобильной электроники БаденВюртембергского объединенного государственного университета. 
UDC 550.834

\title{
PHASE-FREQUENCY DETECTION OF SEISMIC SIGNALS
}

\author{
Alexander I. Kochegurov 1 , \\ kaicc@tpu.ru \\ Viktor Geringer ${ }^{2}$, \\ geringer@dhbw-ravensburg.de \\ 1 National Research Tomsk Polytechnic University, \\ 30, Lenin avenue, Tomsk, 634050, Russia. \\ 2 Baden-Wuerttemberg Cooperative State University, \\ 2, Marienplatz, DHBW-Ravensburg, Germany.
}

Relevance. Currently, in the search for oil and gas fields, much attention is paid to more active involvement of poorly explored territories in exploration, as well as to the study of natural reservoirs in areas of industrial development of deposits. For these purposes, seismic methods are widely used, as a result of which huge data arrays are formed, which are subject to subsequent processing and interpretation. One of the tasks to be solved at the processing stage is reliable detection of seismic signals, this task is especially important when tracking waves, since the quality of construction of reflecting boundaries depends on the reliability of detection. Therefore, the construction of effective methods and algorithms for detecting signals based on the analysis of the phase response, with a higher degree of resistance to interference, is a very urgent task.

The aim of the research is to construct methods for detecting signals based on the optimal and suboptimal processing of the phasefrequency characteristic of seismic waves obtained when searching for oil and gas fields and to study these methods effectiveness on models of seismic pulses by calculating the probability characteristics of detection errors.

Methods and means of research: methods of statistical analysis and synthesis of algorithms, theory of random processes, discrete Fourier transform, methods of processing and interpretation of seismic data, mathematical modeling and computational experiment.

Results. Based on the maximum likelihood method, an optimal procedure for detecting seismic signals by their phase-frequency characteristics is constructed. Analytical expressions are obtained for the probabilities of detection errors. It is shown that optimal phasefrequency detection provides results close to the results of the absolutely optimal signal detection method. For practical implementation of the developed method, suboptimal processing of the phase response (equilibrium processing) is proposed, which does not require information on distribution of signal energy in the frequency domain. The noise immunity of the detection method with equilibrium processing was studied and estimates of losses were obtained depending on the number of used frequency components in the signal spectrum. The place of application of the developed detection methods for solving problems of tracking seismic waves is shown.

\section{Key words:}

Seismic waves, optimal phase-frequency detection, likelihood ratio, equilibrium processing, seismic wave tracking.

\section{REFERENCES}

1. Boganik G.N., Gurvich I.I. Seysmorazvedka [Seismic exploration] Tver, AIS Publ., 2006. 744 p.

2. Leviant V.B., Kvasov I.E., Petrov I.B. Substantiation for the direct detection and mapping of fractured formations based on using scattered compressional waves. Technologies of seismic exploration, 2017, no. 1, pp. 72-89. In Rus.

3. Denisov M.S. Stable adaptive signature deconvolution. Technologies of seismic exploration, 2017, no. 1, pp. 48-55. In Rus.

4. Naveed Iqbal, Entao Liu, McClellan J.H., Abdullatif A. Al-Shuhail Sparse blind deconvolution of seismic data via spectral projectedgradient. IEEE Access, 2019, pp. 1-12. Available at: https://arxiv.org/abs/1611.03754 (accessed 5 March 2020).

5. Silvia M.T., Robinson E.A. Obratnaya filtratsiya geofizicheskikh bremennykh ryadov pri razvedke na neft $i$ gaz. [The return filtration of geophysical temporary ranks at investigation on oil and gas] Moscow, Nedra Publ., 1983. 382 p.

6. Mohamed Mhmod, Feng Xuan, Xu Cong. Parameters effects on spiking deconvolution of land seismic data. Global Geology, 2015, vol. 18 (4), pp. 226-231

7. Romanov V.V. Possibilities of increase in resolution of seismographic records of a method of the refracted waves (MPV). Technologies of seismic exploration, 2013, no. 4, pp. 67-73. In Rus.

8. Stankevich L.S. Obrabotka seysmicheskikh razrezov metodom dinamicheskogo szhatiya [Processing seismic cuts by the method of dynamic compression]. Geofizika, geofizicheskoe priborostroenie, 2015, no. 1 (21), pp. 78-85.
9. Mitrofanov G.M. Homomorphic filtering and deconvolution. Technologies of seismic exploration, 2015, no. 1, pp. 46-56. In Rus.

10. Egbai J.C., Atakpo E., Aigbogun C.O. Predictive deconvolution in seismic data processing in Atala prospect of rivers State, Nigeria. Advances in Applied Science Research, 2012, vol. 3 (1), pp. 520-529.

11. Lindberg D.V., More H. Blind categorical deconvolution in twolevel hidden Markov models. IEEE Transactions on Geoscience and Remote Sensing, 2014, vol. 52, pp. 7435-7447.

12. Idan Ram, Israel Cohen. Multichannel deconvolution of seismic signals using statistical MCMC methods. IEEE Transactions on signal processing, 2010, vol. 58, no. 5, pp. 2757-2770.

13. Kazemi N., Bongajum E., Sacchi M. Surface-consistent sparse multichannel blind deconvolution of seismic signals. IEEE Transactions on geoscience and remote sensing, 2016, vol. 54, pp. 3200-3207.

14. Kochegurov A.I., Ivanchenkov V.P., Orlov O.V. Forecast of geological profile based on detailed processing materials in the software algorithmic complex «Geoseif». Bulletin of the Tomsk Polytechnic University. Geo Assets Engineering, 2019, vol. 330, no. 6, pp. 134-144. In Rus.

15. Kochegurov A.I., Kochegurova E.A., Kozlov A.E., Mongolin A.S. Phase/frequency deconvolution of seismic signals. ISSCS $2019-$ International Symposium on Signals, Circuits and Systems. Proceedings. Iasi, Romania, 2019. New York, IEEE, 2019. pp. 1-4.

16. Pestryakov V.B. Fazovye radiotekhnicheskie sistemy [Phase radio engineering systems]. Moscow, Sov. Radio Publ., 1968. 468 p.

17. Levin B.R. Teoreticheskie osnovy statisticheskoy radiotekhniki [Theoretical foundations of statistical radio engineering]. Moscow, Sov. Radio Publ., 1974. B. 1, 552 p. 
18. Levin B.R. Teoreticheskie osnovy statisticheskoy radiotekhniki [Theoretical foundations of statistical radio engineering]. Moscow, Sov. Radio Publ., 1975. B. 2, 392 p.

19. Poisk, obnaruzhenie $i$ izmerenie parametrov signalov $v$ radionavigatsionnykh sistemakh [Search, detection and measurement of signal parameters in radio navigation systems]. Ed. by Yu.M. Kazarinova. Moscow, Sov. Radio Publ., 1975. 296 p.

20. Kats S.A., Ershova T.N., Shubik B.M. Metodika i rezultaty opredeleniya otnosheniya signal/shum pri registratsii seysmicheskogo polya [Methodology and results of determining

\section{Information about the authors}

Alexander I. Kochegurov, Cand. Sc., associate professor, National Research Tomsk Polytechnic University.

Viktor Geringer, Cand. Sc., Head of the Laboratory of Automotive Electronics Baden-Wuerttemberg Cooperative State University. the signal-to-noise ratio during registration of a seismic field] Prikladnaya geofizika, 1973, Iss. 72, pp. 59-70.

21. Dubinin D., Geringer V., Kochegurov A., Reif K. A stochastic algorithm for generating images by a two-dimensional SemiMarkov Model. at- Automatisierungstechnik, 2014, vol. 62, no. 1, pp. 57-64. In Germ.

Received: 9 April 2020. 